\title{
Variabilité de Pseudocercosporella herpotrichoides (Fron) Deighton, agent du piétin-verse des céréales
}

\author{
P Poupard, N Cavelier
}

INRA, Centre de recherches de Rennes, SRIV BP 2935650 Le Rheu, France

(Reçu le 26 août 1991; accepté le 25 novembre 1991)

\begin{abstract}
Résumé - Pseudocercosporella herpotrichoides, l'agent du piétin-verse des céréales, présente une variabilité intraspécifique mise en évidence par plusieurs critères: pouvoir pathogène, morphologie et physiologie in vitro, profils protéiques et enzymatiques, sensibilité aux fongicides. Une synthèse des données est réalisée concernant ces différents critères. L'ensemble de ces caractères permet la différentiation de 2 groupes de souches au sein de l'espèce: souches à croissance normale ou type $W$ ou $P$ h var herpotrichoides d'une part, souches à croissance lente ou type $\mathrm{R}$ ou $P h$ var acuformis d'autre part. La variabilité à l'intérieur de ces 2 populations est également importante si l'on se base sur ces mêmes critères d'étude. Les divers aspects de la variabilité de l'agent pathogène sont développés de manière à montrer leur intérêt dans la lutte contre cette maladie, tant au niveau de l'utilisation raisonnée de fongicides qu'à celui de la recherche de sources de résistance.
\end{abstract}

morphologie / physiologie / protéine et enzyme/pouvoir pathogène/sensibilité aux fongicides

Summary - Variability of Pseudocercosporella herpotrichoides (Fron) Deighton, the cause of eyespot in cereals: a review. Pseudocercosporella herpotrichoides, the cause of eyespot in cereal crops, shows variability characterized on the basis of pathogenicity, cultural morphology, physiology, protein and isozyme patterns and sensitivity to fungicides. The species herpotrichoides comprises 2 main pathotypes distinguished by these criteria: fast growing colonies (I or N) correlated with $\mathrm{W}$-type and $\mathrm{P} \mathrm{h}$ var herpotrichoides; slow growing colonies (II or L) correlated with R-type and $\mathrm{P}$ h var acuformis. Growth rate (on agar medium), colony morphology and pigmentation, conidial form allow differentiation into 2 groups. Nevertheless, there are considerable differences between individual isolates in both groups and these criteria do not fully confirm the distinction made on the basis of pathogenicity. The first virulence group is characterised by the $W$ type isolates: their pathogenicity is high to wheat and barley, and is low to rye. The second group is characterized by the $R$ type isolates, which are equally pathogenic to wheat, barley and rye. A type is also described which is pathogenic to couch grass. Apart from these types, 1 type was observed which is pathogenic only to wheat and Aegilops squarrosa. All types are pathogenic to Aegilops ventricosa. There are considerable differences in pathogenicity between individual isolates in both the $W$ and the $R$ type groups. Differences in controlled environment conditions may explain the varying results in the studies on pathogenicity of both types. The penetration of the stem by the $W$ type (or $N$, or var herpotrichoides) occurs earlier than that by the $R$ type (or L, or var acuformis), which are isolated more frequently at the end of the season. Both types can exist in the same plant or even on the same lesion; interactions between both types can influence the development of eyespot. Electrophoretic studies show that specific patterns (proteins, isozymes) exist for the $W$ type, $N$ type and var herpotrichoides, and also for the $R$ type, $L$ type and var acuformis. Contrary to the former characteristics, high homogeneity is observed within the same type. $\mathrm{P}$ herpotrichoides isolates can be clearly and more objectively differentiated by biochemical markers. The development of resistance to fungicides has induced different phenotypes which react in a very specific way to 1 type of compound. Sensitive or resistant isolates to benzimidazole, thiophanate and phenylcarbamate compounds are common in both types. Three types are distinguished according to their sensitivity 
to ergosterol biosynthesis inhibitors: la or $\mathrm{Na}$ which are sensitive to $\mathrm{DMI}$, lb or $\mathrm{Nb}$ and $/$ or $L$ which are less sensitive to DMI. Recently, several prochloraz resistant strains (II or $L$ type) have been isolated on winter wheat in France. These different aspects of the variability of the fungus are discussed to show their importance in eyespot control both for the optimal use of fungicides and for research into host resistance sources. The perfect state in which $\mathrm{P}$ herpotrichoides is found, Tapesia yallundae, has only recently been described and its importance is not yet known. It provides a new means for the study of the relationships between isolates types.

morphology/physiology/protein and isozyme patterns/pathogenicity/sensitivity to fungicides

\section{INTRODUCTION}

Les changements de populations de Pseudocercosporella herpotrichoides, agent du piétinverse des céréales, observés au champ ces 10 dernières années en Europe, ont posé de façon aiguë le problème de la variabilité de ce champignon. Deux types de souches sont maintenant couramment isolées, dénommées souches à croissance normale ou rapide et souches à croissance lente. Bien qu'identifiées depuis 1966 par Lange de la Camp (1966a,b), les souches à croissance lente ne sont devenues fréquentes que dans les années 1980. En Europe du Nord (Grande-Bretagne, Nord de l'Allemagne, Belgique, Nord de la France), le phénomène est tel que ce type d'isolats a quasiment remplacé le type préexistant.

La variabilité intraspécifique de $P$ herpotrichoides a déjà fait l'objet de nombreuses études ayant conduit à la caractérisation de types d'isolats selon différents critères: virulence, morphologie et physiologie in vitro et sensibilité aux fongicides. Plus récemment les recherches se sont orientées vers d'autres critères tels que la caractérisation des souches par électrophorèse des protéines et enzymes.

Les observations réalisées, tant au champ qu'en conditions contrôlées incitent à penser que les différentes formes du parasite auraient chacune un comportement épidémiologique propre (Fitt et al, 1988). L'étude de l'épidémiologie de $P$ herpotrichoides a été largement abordée dans la littérature, mais la notion de variabilité du parasite n'est que peu ou même pas introduite (Fehrmann et Schrodter, 1971; Rapilly et al, 1979; Hollins et Scott, 1980; Fitt, 1985), excepté les travaux de Goulds et Fitt (1988, 1990a,b, 1991).

L'utilisation des fongicides en pratique s'accompagne de modifications au sein des po- pulations de $P$ herpotrichoides. Aussi la variabilité de ce parasite est-elle à prendre en compte si l'on veut lutter contre celui-ci de façon efficace et rationnelle. II s'agit d'une part, d'éviter la sélection d'isolats peu sensibles aux produits couramment utilisés sur céréales, dirigés ou non contre ce champignon et, d'autre part, d'adapter la lutte en fonction de la population dominante.

Dans cet article, nous nous proposons de faire la synthèse de résultats obtenus par différentes équipes: dans une première partie, nous aborderons la variabilité de $P$ herpotrichoides au niveau de caractères morphologiques, physiologiques et biochimiques, la seconde partie traitant de la variabilité liée au pouvoir pathogène. Nous envisagerons dans une troisième partie la variabilité de comportement du champignon vis-à-vis de différents fongicides. Nous avons conservé les dénominations des phénotypes de l'espèce $P$ herpotrichoides utilisés par les différents auteurs dans leurs publications et travaux. Ces différentes dénominations sont regroupées dans le tableau I.

\section{VARIABILITÉ DE $P$ HERPOTRICHOIDES BASÉE SUR DES CRITĖRES MORPHOLOGIQUES, PHYSIOLOGIQUES ET BIOCHIMIQUES}

\section{Critères morphologiques et physiologiques}

Lange de la Camp (1966a) semble être la première à s'être interrogée sur la spécialisation à l'intérieur de l'espèce herpotrichoides. Cet auteur a comparé des isolats de diverses provenances sur milieu artificiel à base de malt 
Tableau I. Dénominations des différents types d'isolats de $P$ herpotrichoides en fonction des groupes de virulence, des critères morphologiques, physiologiques et de sensibilité aux fongicides.

\begin{tabular}{lll}
\hline \multicolumn{1}{c}{ Virulence } & \multicolumn{1}{c}{$W$ (weizen wheat) } & \multicolumn{1}{c}{$R$ (roggen-rye) } \\
\hline $\begin{array}{l}\text { Vitesse de croissance } \\
\text { journalière in vitro }\end{array}$ & $\mathrm{N}$ (normale) & $\mathrm{L}$ (iente) \\
$\begin{array}{l}\text { Croissance, morphologie } \\
\text { des colonies in vitro }\end{array}$ & var & var \\
$\begin{array}{l}\text { Morphologie des conidies } \\
\text { Sensibilité à certains }\end{array}$ & herpotrichoides & acuformis \\
inhibiteurs de la biosynthèse & la ou Na (S aux «triazoles » & II ou L (R aux «triazoles \\
des stérols & $\mathrm{R}$ au fenpropimorphe) & (S au fenpropimorphe) \\
& Ib ou Nb (R aux «triazoles $~$ & \\
\hline
\end{tabular}

$S$ : sensibilité; $R$ : moindre sensibilité.

gélosé. Les colonies se différencient selon leur pigmentation, leur morphologie et vitesse de croissance. Différents types d'isolats sont ainsi définis à l'intérieur de 2 groupes:

- le groupe 1 comprend des isolats à colonies blanc-gris ou gris souris à croissance uniforme;

- le groupe 2 des isolats à colonies pour la plupart rose-orangé à croissance irrégulière (marges pennées).

Les principaux types d'isolats dans chacun des groupes sont classés d'après leur vitesse de croissance: les types définis dans le groupe 1 ont une croissance plus rapide que ceux définis dans le groupe 2 et la capacité à sporuler des isolats du groupe 2 semble, en général, plus importante. Ces 2 groupes se différencient également par leur pouvoir pathogène (voir Définition des types d'isolats d'après leur virulence), qui est à l'origine de l'appellation «W» ou weizen (blé en allemand) pour les isolats du premier groupe et « $\mathrm{R}$ » ou roggen (seigle en allemand) pour ceux du second groupe.

Les observations de Scott et al (1975) en Angleterre rejoignent celles de Lange de la Camp. Des isolats particuliers montrent en culture sur milieu PDA des colonies à morphologie inhabituelle avec une croissance plus lente, des marges irrégulières, moins de mycélium aérien, une absence de dôme caractéristique, une pigmentation gris-rose, gris pâle ou gris sombre ainsi qu'une sporulation abondante. Ces isolats particuliers correspondent au groupe 2 (type roggen) défini précédemment: la même dénomination que celle de Lange de la Camp est reprise pour désigner les 2 types d'isolats ainsi définis: «W» (wheat) et «R» (rye).
Les travaux de Nirenberg (1981 et 1984) basés sur la forme des conidies, la vitesse de croissance et l'aspect des colonies in vitro, conduisent à la définition de 2 variétés au sein de l'espèce herpotrichoides: il s'agit des variétés herpotrichoides et acuformis. Deux nouvelles espèces de Pseudocercosporella sont également décrites: aestiva et anguioides. Les critères morphologiques et physiologiques permettant de différencier les variétés herpotrichoides et acuformis sont les suivants:

- le rayon de croissance des colonies sur milieu PDA à $10 \mathrm{j}$ et à $20^{\circ} \mathrm{C}$ est en moyenne de $12 \mathrm{~mm}$ pour herpotrichoides, de $5 \mathrm{~mm}$ pour acuformis. Les températures cardinales de croissance mycélienne sont identiques;

- chez herpotrichoides, les colonies à marges uniformes sont grises ou gris-olive tandis qu'elles sont à marges irrégulières et pigmentées en gris ou marron-gris chez acuformis (sur milieu PDA). Les 2 variétés ne peuvent s'anastomoser entre elles;

- les conidies d'herpotrichoides sont courbes ou droites et mesurent $52 \mu \mathrm{m}$ de longueur moyenne, celles d'acuformis sont toujours droites, longues de $65 \mu \mathrm{m}$ en moyenne. Les 2 variétés sporulent abondamment sur milieu SNA et sous lumière noire, en particulier la variété acuformis.

En Allemagne, Schreiber et Prillwitz (1985) montrent l'existence d'une variabilité à l'intérieur de chacune des variétés herpotrichoides et acuformis. Cette variabilité porte sur la vitesse de croissance et la coloration du mycélien en culture sur milieu PDA.

Creighton (1989), en Angleterre, signale l'existence d'isolats à morphologie intermédiaire sur milieu PDA qui ne correspondraient 
pas forcément à des mélanges d'isolats de chacun des types et dont la description diffère de celle des précédents auteurs. En Belgique, des isolats à morphologie intermédiaire entre types $W$ et $R$ sont également mis en évidence (Maraite et al, 1985).

En France, le principal critère de différenciation des isolats est la vitesse de croissance journalière sur milieu PDA ou sur milieu à base de sels minéraux, glucose et extrait de levure, à $20^{\circ} \mathrm{C}$ : on distingue les isolats à croissance normale ou rapide (dénommés $N$ ou 1) dont la vitesse de croissance est supérieure à $2 \mathrm{~mm}$, de ceux à croissance lente (dénor més $L$ ou II) dont la vitesse de croissance est inférieure à $1,5 \mathrm{~mm}$ par jour (Leroux et Gredt, 1985; Cavelier et al, 1987).

La différenciation des 2 types de souches est également possible par le test de pigmentation du milieu de culture à base de semoule de maïs: les isolats $W$ sont à l'origine d'une coloration vert-noir du milieu, tandis que les isolats $R$ induisent une pigmentation rose ou brun pâle (Creighton, 1989).

Au plan de la physiologie de la nutrition des 2 types d'isolats, quelques données concernant l'influence des sucres sur la croissance du champignon figurent dans la littérature. Bien que la maladie soit qualifiée de high sugar disease (Evans et Rawlinson, 1977), l'augmentation de la teneur en glucose dans le milieu nutritif semble affecter la croissance des souches; cet effet dépressif se faisant plus sentir chez la variété herpotrichoides que chez acuformis. De même, une concentration importante en fructose (de l'ordre de $3 \%$ ) inhibe fortement l'élongation des hyphes des 2 types (Nirenberg, 1984).

Dans un souci de clarification, de nombreux auteurs ont cherché à établir des correspondances entre types décrits selon les différents critères. Nirenberg (1981), King et Griffin (1985) ont associé les variétés herpotrichoides et acuformis respectivement aux types $W$ et $R$ définis auparavant par Lange de la Camp (1966a,b) et Scott et al (1975). En Angleterre, les isolats Fast even et Slow feathery décrits par Brown et al (1984) selon la morphologie et la vitesse de croissance des colonies in vitro sont assimilés aux types $W$ et $\mathbf{R}$ (Hollins et al, 1985). Plus largement, Leroux et al (1985) font la relation entre, d'une part, - type W, variété herpotrichoides et isolat $\mathrm{N}$ ou I- et, d'autre part, -type R, variété acuformis et isolat $\mathrm{L}$ ou II-; toutefois Mauler et
Fehrmann (1987a) affirment que, d'après l'étude morphologique du champignon (caractéristiques des colonies et conidies), la classification en 2 variétés d'après Nirenberg (1981) ne recoupe pas parfaitement celle en 2 groupes de virulence.

\section{Critères biochimiques}

Les études électrophorétiques concernant $P$ herpotrichoides sont toutes récentes (elles ont débuté en 1987). Elles comprennent, non seulement la mise en évidence des profils de protéines totales du mycélium, mais également la révélation de nombreux systèmes enzymatiques.

Les travaux de Bolik et al (1987) montrent qu'il est possible de différencier aisément les espèces herpotrichoides, anguioides et aestiva au sein du genre Pseudocercosporella sur la base des protéines totales en conditions natives ou dénaturantes. En comparant les profils protéiques d'autres espèces de champignons parasites du pied des céréales (Rhizoctonia cerealis, Fusarium sp, Microdochium nivale), des bandes protéiques spécifiques de l'espèce $P$ herpotrichoides ont été observées (Weege et al, 1991). Des bandes protéiques caractéristiques de chaque type de souches sont également mises en évidence (Bolik et al, 1987; Lind et al, 1987; Unger, 1989; Poupard et Cavelier, 1990; Weege et al, 1991); ce nombre de bandes spécifiques varie en fonction des auteurs (1-5 bandes); les différences à ce niveau peuvent provenir du nombre d'isolats étudiés (1-10 isolats de chaque type selon les auteurs) et des conditions électrophorétiques mises en œuvre dans ces différents travaux.

L'étude de Julian et Lucas (1990) met en évidence la difficulté de distinguer les 2 types de souches par leurs protéines solubles totales (nombre élevé de bandes protéiques, médiocre reproductibilité des profils), mais par contre, la différentiation est aisée en révélant certains systèmes enzymatiques, dont les systèmes estérase, glutamate-déshydrogénase, glucose-phosphate-isomérase et malate-déshydrogénase. Sur une centaine d'isolats de $P$ herpotrichoides d'origine géographique différente, aucun n'a montré un profil iso-enzymatique intermédiaire entre type $W$ et type R. La diversité géographique n'a induit aucune variation dans les bandes majeures d'iso-enzymes de chaque type d'isolats: une 
grande homogénéité existe donc à l'intérieur d'un même type. Cette étude de polymorphisme enzymatique est en accord avec la classification de Nirenberg (1981). Quelques isolats à morphologie in vitro de type $\mathrm{W}$ ont cependant été classés dans le type $R$ d'après leurs profils enzymatiques; ces isolats particuliers ont, par ailleurs, un pouvoir pathogène correspondant au type $\mathrm{R}$.

D'autres travaux mettent également en évidence la différentiation des variétés herpotrichoides et acuformis par certains systèmes enzymatiques mais sur un nombre plus limité de souches: les systèmes étudiés sont les estérases mycéliennes et 2 groupes d'enzymes extracellulaires (pectine-méthyl-estérases et ribonucléases) (Unger, 1989; Weege et al, 1991).

Qu'il s'agisse de la séparation des protéines mycéliennes totales ou de la mise en évidence de certains systèmes enzymatiques, il semblerait que les études électrophorétiques puissent permettre de différencier les 2 types de souches de façon plus objective que les critères décrits dans la section Critères morphologiques et physiologiques; la mise en œuvre de ces techniques pourrait se révéler utile en routine pour la caractérisation de $P$ herpotrichoides.

\section{VARIABILITÉ DU POUVOIR PATHOGÈNE DE $P$ HERPOTRICHOIDES}

Nombreuses sont les données dans la littérature qui concernent les interactions entre $P$ herpotrichoides et sa plante hôte; ces dernières sont étudiées la plupart du temps dans le but de sélectionner des génotypes résistants. II nous a semblé intéressant de dégager plusieurs points concernant:

- la gamme d'hôtes et les spécialisations de ce champignon;

- l'incidence des types d'isolats et l'évolution de chaque type en relation avec le développement de la plante;

- la nature des relations entre types d'isolats installés sur la même plante.

\section{Définition des types d'isolats d'après leur virulence}

$P$ herpotrichoides peut être décrit comme un parasite à large gamme d'hôtes; il est patho- gène sur les différentes céréales, le blé, l'orge, le seigle et l'avoine. Il est également capable de se développer sur certaines graminées spontanées dont le chiendent, la fétuque élevée, le paturin, le vulpin des champs (Ponchet, 1959). La sensibilité du seigle et de l'avoine au parasite est toutefois minime (Foex et Rosella, 1934; Hårdh, 1953). L'Aegilops sp, graminée très voisine du blé, présente des différences de sensibilité à ce champignon. Les lignées les moins sensibles d'Aegilops ventricosa sont utilisées dans les programmes de sélection pour la résistance au piétin-verse (Doussinault et Dosba, 1977).

Lange de la Camp (1966a,b) définit 2 groupes de virulence:

- le groupe 1 comprend des isolats, dénommés "W" ou "weizen" issus en majorité de blé et d'orge dont l'agressivité, forte sur blé et orge, est négligeable sur seigle;

- le groupe 2 est constitué par des isolats appelés « $\mathrm{R}$ » ou «roggen» provenant principalement de seigle; leur pouvoir pathogène sur blé, orge et seigle est comparable.

Les observations de Scott et al (1975) confirment celles de Lange de la Camp: in vivo, des isolats particuliers issus de seigle (isolats « $R$ » ou «rye») se différencient également des autres isolats ( $W$ » ou " wheat"), provenant de blé ou d'orge, par leur agressivité sur seigle identique à celle sur blé.

Ces auteurs introduisent la notion de groupes de virulence et non pas de formes spéciales chez $P$ herpotrichoides. II n'y aurait pas d'adaptation particulière du parasite à un hôte particulier, quelle que soit la plante d'origine de l'isolat. Cette remarque est reprise par Schreiber et Prillwitz (1985), mais de façon plus nuancée, avec l'idée d'une certaine spécificité de la variété herpotrichoides sur blé. De même certains isolats issus de seigle et décrits par Scott et al (1975) ont une forte affinité pour cette céréale et seraient moins adaptés à infecter le blé et l'orge.

Cunningham (1981) décrit un type supplémentaire (différent du type R), le type $C$ («couch»), et l'oppose au type W. Le pouvoir pathogène du type $C$, en plus de celui exprimé sur blé et sur orge, s'étend au chiendent.

Scott et Hollins (1980) résument les intéractions existant entre types d'isolats et hôtes différentiels: à côté des types $W, R$ et $C$, un type supplémentaire dont l'agressivité est observée seulement sur blé et Aegilops squarrosa est décrit. Les types $\mathrm{R}$ et $\mathrm{C}$ sont 
également pathogènes sur cette dernière graminée tandis qu'aucun des types n'attaque Aegilops ventricosa.

\section{Pouvoir pathogène des différents types de souches observé sur plantule et plante adulte}

Les remarques concernant ce point sont sujettes à controverse : elles reflètent les résultats d'essais in vivo qui ne sont pas forcément réalisés dans les mêmes conditions (inoculum, support végétal, facteurs environnementaux). Des tendances peuvent toutefois être dégagées quant à l'agressivité comparée des 2 types de souches:

Ainsi, sur plantules de blé, le type $W$ est-il en général plus pathogène que le type $R$, quel que soit le type d'inoculum (conidies, mycélium) (Fitt et al, 1987; Creighton et al, 1989). Hollins et al (1985) obtiennent des résultats n'allant pas dans ce sens, tandis que Brown et al (1984) n'observent pas de différences significatives entre les pouvoirs pathogènes de chaque type. Les souches $W$ sont plus agressives sur blé que sur seigle, tandis que les souches $\mathrm{R}$ ont la même agressivité sur blé et sur seigle (Lange de la Camp, 1966b; Scott et al, 1975; Hollins et al, 1985). Des études plus récentes mettent en évidence que les 2 types d'isolats sont plus agressifs sur blé que sur seigle. Sur seigle, le pouvoir pathogène du type $\mathrm{R}$ est plus important que celui du type W (Mauler et Fehrmann 1987a; Creighton et al, 1989). Ces différents travaux ne sont pas réalisés dans les mêmes conditions de température (entre 7 et $15^{\circ} \mathrm{C}$ selon les auteurs), or il semblerait que ce facteur joue un rôle déterminant sur l'agressivité des souches (Lange de la Camp, 1966b; Goulds et Fitt, 1991). Les travaux de Mauler et Fehrmann (1987a) et de Creighton et al (1989) remettent néanmoins en question l'opportunité de l'appellation des types de souches en fonction de leur virulence. Sur plantule d'orge, la différence de pouvoir pathogène entre type $W$ et type $\mathbf{R}$ n'est pas distincte (Mauler et Fehrmann, 1987a).

Le pouvoir pathogène des différents types d'isolats déterminé sur plantule ne reflète pas toujours celui exprimé sur plante adulte (Higgins et Fitt, 1985).

Au stade plante adulte et en conditions contrôlées, l'agressivité des isolats $R$ ou $L$ sur tiges de blé est inférieure à celle des isolats
W ou N (Higgins et Fitt, 1985; Cavelier et al, 1988). Au champ, Bateman et al (1986) observent un pouvoir pathogène qui est supérieur pour le type $\mathrm{R}$ sur blé d'hiver. Ce dernier résultat demeure toutefois très ponctuel.

\section{Relation entre l'évolution des deux types d'isolats et le développement de la plante hôte}

Les remarques qui suivent constituent la synthèse de résultats d'essais en conditions contrôlées et de plein champ: ont été étudiées, d'une part, la progression du parasite dans la plante à travers les gaines jusqu'à la tige et, d'autre part, la relation entre le développement de la plante et l'intensité de la maladie provoquée par chacun des types.

Dans les conditions naturelles du Palatinat en Allemagne, Schreiber et Prillwitz (1985) isolent de façon importante la variété acuformis à tous les stades étudiés (21-25: tallage, 29-30: fin tallage-redressement, 51-59: gaine éclatée-épi dégagé) avec une augmentation de ce type d'isolat à tous les stades.

En France, il est possible d'isoler les 2 types à tous les stades de la plante (l'isolement d'un type déterminé est plus en rapport avec l'origine géographique de l'échantillon végétal); de façon générale, le type $L$ est également isolé plus fréquemment en fin de végétation de la céréale (Leroux et Gredt, 1988b).

En ce qui concerne le passage à travers les gaines, en conditions contrôlées sur blé, Schreiber et Prillwitz (1985) observent un développement semblable des 2 variétés jusqu'à la septième gaine, puis à partir de cette dernière, une évolution plus rapide de la variété herpotrichoides.

Mauler et Fehrmann $(1987 a, b)$ en étudiant les différents stades de l'infection sur différentes variétés de blé ont remarqué que, quelle que soit la sensibilité de la céréale, les processus de l'infection liés au type $R$ sont plus lents que ceux dus au type $W$ et ne conduisent pas au niveau d'attaque élevé engendré par ce dernier type.

Cavelier et al (1987) montrent que l'agressivité du type $\mathrm{N}$ est supérieure à celle du type $\mathrm{L}$ à la montaison: au stade 39 , le type $\mathrm{N}$ est présent sur la tige, contrairement au type $\mathrm{L}$. La fréquence des isolats de type $L$ observée sur gaines est toujours voisine de celle de l'inoculum de départ, alors qu'au moment du 
passage sur tige, cette fréquence est généralement inférieure. A la maturation, l'intensité des attaques peut être cependant identique pour les 2 types d'isolats.

En Belgique, il n'a pas été observé de différence de pénétration à travers les gaines entre les variétés herpotrichoides et acuformis, cependant le passage de la dernière gaine à la tige est plus lent pour la variété acuformis; la section nécrosée provoquée par la variété herpotrichoides à la maturation est plus importante que celle causée par la variété acuformis (Van Schingen et al, 1989; Moreau et al, 1990).

Sur orge, la différence d'évolution entre les 2 types d'isolats est moins distincte. Sur seigle, les symptômes sont d'intensité identique pour les 2 types jusqu'à la cinquième gaine; seule la variété acuformis parvient sur la huitième gaine (Schreiber et Prillwitz, 1985; Mauler et Fehrmann, 1987a).

Des essais de contamination des différents types d'isolats à différents stades de la plante montrent les résultats suivants: sur blé, l'attaque provoquée par le type $W$ est plus importante si la contamination a lieu à des stades plus précoces (13-31) que tardifs (3739 ); le type $R$ entraîne moins de dégâts que le type $W$ pour une contamination réalisée au stade 13. L'intensité de maladie est identique pour les 2 types en contaminant au stade 31 (Mauler et Fehrmann, 1987b).

À différentes dates de contamination (aux stades 3 feuilles, tallage ou début montaison), il n'y a pas de différence de pouvoir pathogène observé entre type $\mathrm{N}$ et type $\mathrm{L}$ à la maturation. Cependant, plus l'inoculum de type L est apporté tardivement, plus le pouvoir pathogène de ce type d'isolats diminue (Cavelier et al, 1987).

\section{Pouvoir pathogène d'une population en mélange}

Dans la nature, il n'est pas rare que les 2 types d'isolats coexistent sur la même plante hôte, voire au sein d'une même lésion (Leroux et Gredt, 1988b); face à ces situations de mélange d'isolats, il semble intéressant de préciser la nature des intéractions entre les types d'isolats in vivo, celle-ci pouvant expliquer en partie l'évolution des populations. II s'agit là encore de résultats d'essais biologiques en environnement artificiel ou naturel.
Dans certains essais réalisés en conditions contrôlées, le mélange des 2 types d'isolats en proportions équivalentes montre un pouvoir pathogène inférieur à celui de chacun des isolats considéré séparément. Ce phénomène est observable sur jeune plante comme sur plante adulte. Au stade plantule, le pouvoir pathogène du mélange d'isolats peut toutefois atteindre celui du type L. L'agressivité d'un inoculum mixte (type $\mathrm{N}$ apporté à un stade donné de la plante suivi du type $L$ apporté à un stade ultérieur) est moindre que celle du type N. Dans ce cas, les lésions des plantes contaminées par le mélange des 2 types d'isolats sont principalement causées par le type $\mathrm{L}$. Ce dernier résultat tend à montrer que le type $L$ gênerait le développement du type $N$. Un isolat de type L est capable de se développer en présence d'un isolat de type $\mathrm{N}$ sauf si la proportion de l'isolat $L$ est très faible dans l'inoculum de départ (Cavelier et al, 1987 et 1990b).

En conditions de plein champ, sur plante contaminée par le mélange des 2 types d'isolats, Moreau et al (1990) observent au stade maturation une section nécrosée intermédiaire entre celles provoquées par un isolat herpotrichoides et un isolat acuformis, tandis que le développement du mélange d'isolats sur gaines est similaire à celui des 2 types d'isolats considérés séparément.

Dans les conditions naturelles, la variété herpotrichoides peut s'installer sur le blé et l'orge déjà contaminés par la variété acuformis mais pas sur le seigle. La variété acuformis s'installe en présence d'herpotrichoides quelle que soit la plante hôte (Schreiber et Prillwitz, 1985).

\section{VARIABILITÉ DE $P$ HERPOTRICHOIDES VIS-Ä-VIS DE FONGICIDES}

Les traitements fongicides contre le piétinverse se sont généralisés avec l'intensification des cultures de blé en Europe de l'Ouest et du Nord dans les années 1970. Les produits du groupe des benzimidazoles et thiophanates se sont d'abord montrés les plus efficaces, puis des souches résistantes à ce type de produit sont apparues; il s'agissait du premier cas de résistance en culture de céréales. Par la suite, d'autres produits ont été utilisés: un imidazole, le prochloraze et des triazoles. 
Les isolats de $P$ herpotrichoides ne sont pas sensibles de la même façon aux différentes molécules utilisées, de plus l'acquisition de résistance a induit un certain nombre de phénotypes qui réagissent de façon spécifique à un type de molécule bien précis. II s'agit là d'une variabilité ayant des conséquences pratiques importantes mais qui peut également servir de marqueur dans l'étude de l'évolution des populations du parasite.

\section{Variabilité de $\mathrm{P}$ herpotrichoides et sensibilité aux fongicides benzimidazoles et thiophanates}

L'apparition des souches résistantes aux benzimidazoles et thiophanates au début des années 1980 a suscité la mise en ceuvre de nombreuses recherches dans les différents pays européens concernés par ce problème, susceptible de conséquences économiques importantes. Les études se sont ainsi orientées vers la caractérisation in vitro des souches résistantes, ainsi que vers le comportement in vivo de populations constituées de souches résistantes. Celles-ci ont pour principal objectif de préciser l'évolution de la résistance en conditions naturelles de façon à limiter son développement et proposer une alternative à l'utilisation de telles matières actives.

\section{Sensibilité in vitro de $P$ herpotrichoides aux fongicides antimitotiques}

L'étude in vitro de la résistance des isolats aux fongicides antiomitotiques indique qu'à ce niveau la variabilité chez $P$ herpotrichoides est importante (Leroux et Cavelier, 1983; Brown et al, 1984; Bateman et al, 1985; Hocart et al, 1990). Sept phénotypes notés de $A$ à $G$ sont en effet décrits en fonction des niveaux de résistance aux benzimidazoles et $\mathrm{N}$-phénylcarbamates. Seul le type A est sensible au carbendazime, tandis que les types $B, C, D$ et $E$ sont fortement résistants et les types $F$ et $G$ faiblement résistants à cette matière active. La résistance croisée positive est toujours observée entre le carbendazime, le bénomyl, le thiabendazole et le thiophanateméthyl.

Les phénotypes $B$ et $C$ présentent une sensibilité accrue aux phénylcarbamates, comparativement au type $A$, résistant : il existe donc une résistance croisée négative entre les benzimidazoles et les phénylcarbamates. Les types $D, E, F$ et $G$ ne sont pas concernés par ce type de résistance (ils sont en outre fortement résistants au diéthofencarbe, fongicide de la famille des phénylcarbamates). Notons enfin que les types A, B, C, D et E sont les plus communs parmi les isolats provenant de blé tendre d'hiver et que tous ces phénotypes décrits (de $A$ à $G$ ) existent au sein de chaque type d'isolat I ou $\mathrm{N}$ et II ou L (Leroux et al, 1985; Leroux et Gredt, 1987, 1988a).

\section{Pouvoir pathogène et capacité de développement comparés des souches sensibles et résistantes aux fongicides benzimidazoles et thiophanates.}

Les études comparatives du comportement de souches sensibles et résistantes ont été entreprises dans le but de connaître l'importance de ce phénomène tant au plan du pouvoir pathogène des différents isolats qu'à celui de sa persistance.

Le pouvoir pathogène sur blé d'un isolat résistant est comparable à celui d'un isolat sensible du même type de virulence que le premier (Brown et al, 1984; Cavelier et Le Page, 1985; Creighton et al, 1989).

La capacité de survie des souches résistantes de phénotypes $B$ et $C$ définis par Leroux et al (1985) semble supérieure à celle des souches sensibles. Après contamination de plantes par le mélange en proportions variables de 2 isolats, l'un sensible et l'autre résistant aux benzimidazoles et thiophanates, la fréquence de la souche résistante après passage sur la plante hôte est toujours supérieure à la fréquence existant dans l'inoculum de départ (Leroux et al, 1985; Cavelier et Le Page, 1985; Cavelier et al, 1985, 1988).

\section{Relations entre les types de sensibilité aux benzimidazoles et thiophanates et les types d'isolats caractérisés par leur virulence, leur physiologie ou morphologie in vitro}

Quel que soit le type d'isolat concerné, l'utilisation de benzimidazoles et thiophanates conduit à la sélection d'isolats résistants ( $\mathrm{Ba}$ teman et al, 1985). Les isolats résistants aux benzimidazoles et thiophanates appartiennent aux 2 types d'isolats, mais un plus grand nom- 
bre d'isolats résistants sont du type acuformis (Sanders et al, 1986), King et Griffin (1985), Hoare et al (1986), Creighton et Bateman (1988) constatent également que la résistance est plus fréquente chez le type $R$ que chez le type $W$. Plusieurs équipes anglaises émettent l'hypothèse que les traitements à base de benzimidazoles et thiophanates sélectionnent les isolats résistants à ces matières actives et les isolats de type $R$, mais la sélection de ces 2 caractères se fait de façon indépendante (Hollins et al, 1985; Bateman et al, 1985; Hoare et al, 1986; Coskun et al, 1987).

\section{Variabilité de $\mathrm{P}$ herpotrichoides en fonction de la sensibilité des isolats aux inhibiteurs de la biosynthèse des stérols}

Des fongicides inhibiteurs de la biosynthèse des stérols sont utilisés en remplacement des benzimidazoles et thiophanates dans la lutte contre le piétin-verse: il s'agit d'inhibiteurs de la $\mathrm{C}$-14 déméthylase appartenant au groupe des imidazoles (prochloraze) ou à celui des triazoles (flusilazole). Pour cette catégorie de produits, on ne peut parler véritablement de résistance; cependant la sensibilité réduite de certains isolats à ces matières actives permet de les subdiviser en différents types.

\section{Sensibilité in vitro des isolats aux inhibiteurs de la biosynthèse des stérols}

En France, 3 types d'isolats sont distingués en fonction de leur sensibilité à certains inhibiteurs de la biosynthèse des stérols, sensibilité aux triazoles en particulier. Le type $\mathrm{Na}$ ou la est sensible in vitro aux inhibiteurs de la biosynthèse des stérols: les $\mathrm{Cl}_{50}$ "mycélium " varient en fonction des molécules étudiées, elles sont en moyenne de $0,05 \mathrm{mg} / \mathrm{l}$ et de $0,2 \mathrm{mg} / \mathrm{l}$ pour le flusilazole et le cyproconazole respectivement. Les types $\mathrm{Nb}$ ou $\mathrm{lb}$ et L ou II sont peu sensibles à de nombreux inhibiteurs de la C-14 déméthylase dont les triazoles (on observe une résistance croisée pour tous les triazoles): les $\mathrm{Cl}_{50}$ «mycélium» visà-vis du flusilazole et du cyproconazole sont respectivement de 0,7 et $9 \mathrm{mg} / \mathrm{l}$ pour le type $\mathrm{Nb}$ et de 0,6 et $8 \mathrm{mg} / \mathrm{l}$ pour le type L; les types $\mathrm{Nb}$ et $\mathrm{L}$ demeurent toutefois sensibles à certains composés comportant l'hétérocycle imidazole (Leroux et Gredt, 1988a). Parmi les isolats de type $\mathrm{Nb}$, il est possible de différencier 2 catégories, les types $\mathrm{Nb}_{1}$ et $\mathrm{Nb}_{2}$ dont les $\mathrm{Cl}_{50}$ "mycélium " vis-à-vis du triadiménol sont respectivement inférieures et supérieures à $10 \mathrm{mg} / \mathrm{l}$ (Leroux et Gredt, 1985). Récemment des souches de type II ou L, notées IIp résistantes au prochloraze ont été isolées: leurs niveaux de résistance sont de l'ordre de 2030 . Ces souches peuvent présenter une sensibilité réduite (ex: flusilazole) ou accrue (ex: cyproconazole, triadiménol) vis-à-vis d'autres inhibiteurs de la C-14 déméthylation des stérols, comparativement au type II ou L, sensible au prochloraze (Leroux et Marchegay, 1991).

Les inhibiteurs de la $\Delta 14$ réductase ou de la $\Delta 7 \rightarrow \triangle 8$ isomérase, tel que le fenpropimorphe, sont moins actifs sur les isolats de type $\mathrm{L}$ ou II que sur les isolats de type $\mathrm{N}$ ou $\mathrm{I}$, contrairement à ce que l'on observe avec le tridémorphe (Leroux et al, 1988).

\section{Comportement in vivo des inhibiteurs de la biosynthèse des stérols sur les populations de $P$ herpotrichoides}

En conditions contrôlées, comme en plein champ, les 2 types d'isolats sont sensibles à l'application de prochloraze, quelle que soit la date de traitement après la contamination. Le type $L$ est peu sensible aux triazoles (flusilazole, tébuconazole). Par rapport à la date de contamination, seule l'utilisation précoce de flusilazole permet la maitrise de ce type d'isolat. Le flusilazole est le plus efficace des triazoles actuels sur les isolats $\mathrm{Na}$, mais son activité peut décroître en cas d'application tardive après la contamination.

Un inoculum composé par le mélange des 2 types d'isolats (type $\mathrm{Na}$ et type $\mathrm{L}$ ) en proportions égales, a un comportement proche de celui du type $\mathrm{Na}$, ceci quel que soit le fongicide apporté (prochloraze, flusilazole). Les traitements à base de triazoles déplacent l'équilibre existant entre les 2 types d'isolats dans un sens favorable à l'installation du type L (Cavelier et al, 1987 et 1988; Cavelier, 1988). En Belgique, dans le cas de population en mélange, Van Schingen et al (1989) observent également un phénomène de sélection des isolats acuformis en présence de traitement à base de flusilazole.

La sensibilité in vivo aux triazoles (flusilazole, cyproconazole) des isolats de type $\mathrm{Nb}$ 
est semblable à celle des isolats de type $L$, elle est donc nettement plus faible que celle des isolats de type $\mathrm{Na}$. Sur les mélanges d'isolats en proportions équivalentes, le flusilazole a une activité réduite sur l'inoculum mixte constitué d'un isolat $L$ et d'un isolat $\mathrm{Nb}$, alors que son activité est bonne sur un isolat $\mathrm{Na}$ associé à un isolat $\mathrm{L}$ ou $\mathrm{Nb}$ (Cavelier et al, 1990a).

\section{CONCLUSION}

Présent dans les régions tempérées, $P$ herpotrichoides est un champignon qui possède une grande variabilité. L'étude de cette dernière a conduit à différents types de caractérisation s'appuyant sur la morphologie, la physiologie, la virulence ou la sensibilité aux fongicides. Malgré le rapprochement établi entre type $\mathrm{W}, \mathrm{N}$ ou I et variété herpotrichoides d'une part, type R, L ou II et variété acuformis d'autre part, le recoupement entre ces différentes dénominations n'est pas parfait (présence d'isolats à morphologie in vitro ne correspondant pas au groupe de virulence, par exemple). Les 2 variétés décrites par $\mathrm{Ni}$ renberg (1981) ne recouvrent pas toutes les typologies existantes (présence d'isolats de type intermédiaire). Les contradictions observées dans les résultats concernant la virulence des souches indiquent que ce critère lourd à mettre en œuvre est difficilement utilisable pour la classification des types d'isolats; cependant il reste valable si l'on étudie le pouvoir pathogène des souches à $10^{\circ} \mathrm{C}$ sur du seigle sélectionné sensible (Doussinault, comm pers).

L'étude de la variabilité protéique et enzymatique du champignon quant à elle, est une voie qui a déjà donné des résultats intéressants. Néanmoins, d'autres méthodes de caractérisation plus fines de l'agent pathogène, en particulier celles permettant une approche moléculaire, sont envisageables. Une étude récente de morphologie et d'ultrastructure in vivo des 2 types de souches observés en microscopie électronique met également en évidence des différences entre types (Daniels et al, 1991).

La mise au point de méthodes de protection raisonnée passe par l'utilisation de cultivars résistants, par une meilleure utilisation donc une meilleure connaissance de l'activité des fongicides. La recherche des sources de résistance a déjà permis d'obtenir des géniteurs possédant un bon niveau de résistance à partir d'Aegilops ventricosa (Doussinault et al, 1983; Jahier et al, 1989). Nous avons vu que $P$ herpotrichoides présentait une certaine variabilité en fonction de sa sensibilité aux molécules fongicides, mais qu'également l'application de certaines matières actives provoquait un déplacement d'équilibre des populations du parasite. Une caractérisation précoce des différents types d'isolats permettrait d'adapter au mieux les traitements fongicides. La mise en œuvre de techniques de détection du champignon, telle que le test ELISA, peut permettre d'obtenir des informations plus précises; il est intéressant, en particulier, de détecter le champignon de façon précoce en l'absence de symptômes, de quantifier éventuellement le mycélium présent dans la plante et de déterminer ensuite un seuil de nuisibilité (Smith et al, 1990). Là encore, des méthodes de détection plus sensibles que les techniques sérologiques, c'est-à-dire celles ayant pour support l'ADN ou l'ARN du champignon seraient d'un intérêt certain. L'amplification enzymatique in vitro de I'ADN ou PCR (polymerase chain reaction) est actuellement à l'étude pour la détection de divers agents pathogènes (Bertheau et Tempe, 1990) et pourrait être appliquée au "modèle " piétin-verse. Des travaux sont actuellement en cours au laboratoire et devraient permettre la mise du point de sondes moléculaires spécifiques de chaque type d'isolat utilisables en détection.

La forme parfaite de $P$ herpotrichoides, Tapesia yallundae Wallwork et Spooner (1988) a été observée pour la première fois en Australie en 1986 (Wallwork, 1987). Depuis, elle a été également signalée en Nouvelle-Zélande (Sanderson et King, 1988) et plus récemment en Europe (Hunter, 1989; King, 1990). Actuellement, son importance pratique n'est pas connue. Les ascospores ont certainement une capacité de dissémination plus importante que les conidies. Moreau et al, (1989) ont montré que les isolats provenant des ascospores d'une même apothécie peuvent être les uns sensibles, les autres résistants au bénomyl. $T$ yallundae pourrait servir de support d'études concernant la génétique de la virulence et celle de la résistance aux fongicides, et donc permettre d'aborder d'une autre manière la variabilité existant chez cet agent pathogène. 


\section{RÉFÉRENCES}

Bateman GL, Smith C, Creighton NF, Li KY, Hollomon DW (1985) Characterization of wheat eyespot populations before development of fungicide resistance. Trans $\mathrm{Br}$ Mycol Soc 85, 335384

Bateman GL, Fitt BDL, Creighton NF, Hollomon DW (1986) Seasonal changes in populations of Pseudocercosporella herpotrichoides (eyespot) in wheat crops. In: Proc Br Insectic Fungic Conf Brighton 2, 441-446

Bertheau Y, Tempe J (1990) L'amplification enzymatique in vitro d'ADN, ou PCR: une technique d'avenir pour la détection des agents phytopathogènes? Phytoma 423, 39-43

Bolik M, Casper R, Lind V (1987) Einsatz serologischer und gelelektrophotetischer Verfahren zum Nachweis von Pseudocercosporella herpotrichoides. Z Pflanzenkr Pflanzenschutz 94, 449-456

Brown MC, Taylor GS, Epton HAS (1984) Carbendazim resistance in the eyespot pathogen $P$ seu docercosporella herpotrichoides. Plant Pathol 33, 101-111

Cavelier N (1988) Variability of the sensitivity of different isolates of Pseudocercosporella herpotrichoides to sterol biosynthesis inhibitor fungicides. In: Cereal breeding related to integrated cereal production. Proc Conf Cereal Sect, Eucarpia Wageningen, 140-144

Cavelier N, Le Page D (1985) Caractéristiques de souches de Pseudocercosporella herpotrichoides (Fron) Deighton, agent du piétin verse des céréales, résistantes aux fongicides benzimidazoles et thiophanates: pouvoir pathogène, capacité de développement. Premières Journ Études Maladies Plant Ann ANPP t 1, 49-55

Cavelier N, Leroux P, Hanrion M, Cure B (1985) Résistance de Pseudocercosporella herpotrichoides aux benzimidazoles et thiophanates chez le blé d'hiver en France. Bull OEPP 15, 495-502

Cavelier N, Rousseau M, Le Page D (1987) Variabilité de Pseudocercosporella herpotrichoides, agent du piétin-verse des céréales: comportement in vivo de deux types d'isolats et d'une population en mélange. $Z$ Pflanzenkr Pflanzenschutz 94, 590-599

Cavelier N, Creighton NF, Prunier M (1988) Variabilité intraspécifique de Pseudocercosporella herpotrichoides, agent du piétin-verse des céréales: pouvoir pathogène de deux types de souches. In: $2^{e}$ Conf Int Maladies Plant Bordeaux. Ann ANPP, 227-235

Cavelier N, Lucas E, Prunier M (1990a) Sensibilité de différents isolats de Pseudocercosporella herpotrichoides à des fongicides inhibiteurs de la biosynthèse de l'ergostérol. Meded Fac Landbouwwet Rijksuniv Gent 55, 989-995

Cavelier N, Poupard P, Lucas E (1990b) Relations between two types of isolates of Pseudocercosporella herpotrichoides, the cause of eyespot of cereals. In: Biotic interactions and soil-borne diseases. $1^{\text {er }}$ Congr Soc Eur Phytopathol, Wageninger, 147-153

Coskun H, Bateman GL, Hollomon DW (1987) Changes in population structure of carbendazim resistant eyespot in wheat and barley between spring and summer 1984. Trans Br Mycol Soc 85, 335-384

Creighton NF (1989) Identification of W-type and R-type isolates of Pseudocercosporella herpotrichoides. Plant Pathol 38, 484-493

Creighton NF, Bateman GL (1988) Improved diagnosis of eyespot pathotypes: application of a new method. In: Brighton Crop Protec Conf Pests and Diseases 8C-27, 1023-1027

Creighton NF, Cavelier N, Fitt BDL (1989) Pathogenicity to wheat and rye of Pseudocercosporella herpotrichoides isolates from France and the UK-Mycol Res 92, 13-17

Cunningham PC (1981) Occurrence, role and pathogenic traits of a distinct pathotype of Pseudocercosporella herpotrichoides. Trans $\mathrm{Br} \mathrm{Mycol}$ Soc 76, 3-15

Daniels A, Lucas JA, Peberdy JF (1991) Morphology and ultrastructure of $W$ and $R$ pathotypes of Pseudocercosporella herpotrichoides on whear seedlings. Mycol Res 95, 385-397

Doussinault G, Dosba $F$ (1977) An investigation into increasing the variability for resistance to eyespot in wheat. Z Pflanzenzücht 79, 122-133

Doussinault G, Dosba F, Jahier J (1983) New results on the improvement of the level of resistance to eyespot in wheat. In: Proc 6th int Wheat Genet Symp, Kyoto, 193-198

Evans ME, Rawlinson CJ (1977) Stem sugars: a possible factor affecting the resistance of wheat to Pseudocercosporella herpotrichoides. Phytopatol Z 89, 37-43

Fehrmann H, Schrödter H (1971) Okologische Untersuchungen zur Epidemiologie von Cercosporella herpotrichoides I Die jahreszeitliche Abhängigkeit von Weizeninfektionen im Freiland. Phytopathol Z 74, 161-174

Fitt BDL (1985) Factors affecting the development of eyespot (Pseudocercosporella herpotrichoides) lesions in wheat. Z Pflanzenkr Pflanzenschutz 92, 455-463

Fitt BDL, Creighton NF, Bateman GL (1987) Pathogenicity to wheat seedlings of wheat-type and rye-type isolates of Pseudocercosporella herpotrichoides. Trans $\mathrm{Br} M y c o l$ Soc 88, 149155

Fitt BDL, Goulds A, Polley RW (1988) Eyespot (Pseudocercosporella herpotrichoides) epide- 
miology in relation to prediction of disease severity and yield loss in winter wheat - a review. Plant Pathol 37, 311-328

Foex E, Rosella E (1934) Étude sur les piétins des céréales. CR Séances Acad Agric Fr 20, 480483

Goulds A, Fitt BDL (1988) The comparative epidemiology of eyespot (Pseudocercosporella herpotrichoides) types in winter cereal crops. In: Pests and Diseases. Brighton Crop Protec Conf, 1035-1040

Goulds A, Fitt BDL (1990a) Effects of fungicide timing on the severity of eyespot on winter wheat or winter barley inoculated with $W$-type or $R$ type isolates of Pseudocercosporella herpotrichoides. Crop Protect 9, 265-270

Goulds A, Fitt BDL (1990b) The development of eyespot on seedling leaf sheaths in winter wheat and winter barley crops inoculated with W-type or R-type isolates of Pseudocercosporella herpotrichoides. J Phytopathol 130, 161173

Goulds A, Fitt BDL (1991) Prediction of eyespot severity on winter wheat or winter barley inoculated with W-type or R-type isolates of Pseudocercosporella herpotrichoides. I Phytopathol 132, 105-115

Hårdh JE (1953) Kevätvehnan Kahutähkaisyydestä sekä sen syista Suomessa. Valtion Maataloousk. Julk 140

Higgins S, Fitt BDL (1985) Pathogenicity of Pseudocercosporella herpotrichoides isolates to wheat seedlings and adult plants. Z Pflanzenkr Pflanzenschutz 92, 176-185

Hoare FA, Hunter T, Jordan VWL (1986) Influence of spray programmes on development of fungicide resistance in the eyespot pathogen of wheat, Pseudocercosporella herpotrichoides. Plant Pathol 35, 506-511

Hocart MJ, Lucas JA, Peberdy JF (1990) Resistance to fungicides in field isolates and laboratory induced mutants of Pseudocercosporella herpotrichoides. Mycol Res 94, 9-17

Hollins TW, Scott PR (1980) Epidemiology of eyespot (Pseudocercosporella herpotrichoides) on winter wheat, with particular reference to the period of infection. Ann Appl Biol 95, 19-29

Hollins TW, Scott PR, Paine JR (1985) Morphology, benomyl resistance and pathogenicity to wheat and rye of isolates of Pseudocercosporella herpotrichoides. Plant Pathol 34, 272-283

Hunter T (1989) Occurrence of Tapesia yallundae, teleomorph of Pseudocercosporella herpotrichoides, on unharvested wheat culms in England. Plant Pathol 38, 598-603

Jahier J, Tanguy AM, Doussinault G (1989) Analysis of the level of eyespot resistance due to genes transferred to wheat from Aegilops ventricosa. Euphytica 44, 55-59
Julian AM, Lucas JA (1990) Isozyme polymorphism in pathotypes of Pseudocercosporella herpotrichoides and related species from cereals. Plant Pathol 39, 178-190

King AC (1990) First record of Tapesia yallundae as the teleomorph of Pseudocercosporella herpotrichoides var acuformis, and its occurrence in the field in the Federal Republic of Germany. Plant Pathol 39, 44-49

King JE, Griffin MJ (1985) Survey of benomyl resistance in Pseudocercosporella herpotrichoides on winter wheat and barley in England and Wales in 1983. Plant Pathol 34, 272-283

Lange de la Camp M (1966a) Die Wirkungsweise von Cercosporella herpotrichoides Fron, dem Erreger der Halmbruchkrankheit des Getreides 1. Festellung der Krankheit. Beschaffenheit und Infektionsweise ihres Erregers. Phytopathol $Z$ $55,34-66$

Lange de la Camp M (1966b) Die Wirkungsweise von Cercosporella herpotrichoides Fron, dem Erreger der Halmbruchkrankheit des Getreides II. Aggressivität des Erregers. Phytopathol Z 56, 155-190

Leroux P, Cavelier N (1983) Caractéristiques des souches de Pseudocercosporella herpotrichoides, agent du piétin-verse des céréales résistantes aux fongicides benzimidazoles et thiophanates. Déf Vég 222, 231-237

Leroux P, Gredt M (1985) Variabilité de la sensibilité de Pseudocercosporella herpotrichoides, agent du piétin-verse des céréales, à des inhibiteurs de la biosynthèse des stérols. $C R$ Séances Acad Sci Paris Sér III 301, 785-788

Leroux P, Gredt M (1987) Caractéristiques de souches de Pseudocercosporella herpotrichoides, agent du piétin-verse des céréales résistantes aux carbendazime et thiabendazole; C R Séances Acad Sci Paris Sér IIIt 305, 395398

Leroux P, Gredt M (1988a) Caractérisation des souches de Pseudocercosporella herpotrichoides, agent du piétin-verse des céréales résistantes à des substances antimitotiques et à des inhibiteurs de la biosynthèse des stérols. Agronomie 8, 719-729

Leroux P, Gredt M (1988b) Résistance de Pseudocercosporella herpotrichoides, agent du piétin-verse des céréales, vis-à-vis de produits antimicrotubules et d'inhibiteurs de la biosynthèse des stérols. In: $2^{e}$ Conf Int Maladies Plant Bordeaux. Ann ANPP, 1313-1320

Leroux P, Marchegay P (1991) Caractérisation des souches de Pseudocercosporella herpotrichoides, agent du piétin-verse des céréales, résistantes au prochloraze, isolées en France sur blé tendre d'hiver. Agronomie 11 767-776

Leroux P, Gredt M, Van Berkel M, Cavelier N, Le Page D (1985) Caractéristiques des souches de 
Pseudocercosporella herpotrichoides isolées en France sur blé d'hiver. Fungic Crop Protec BCPC Monograph 31, 371-374

Leroux P, Gredt M, Boeda P (1988) Resistance to inhibitors of sterol biosynthesis in field isolates or laboratory strains of the eyespot pathogen Pseudocercosporella herpotrichoides. Pestic Sci $23,119-129$

Lind V, Casper R, Bolik M, Zuchner S (1987) Die Anwendung einer serologischen Methode zum Nachweis des Befall mit Pseudocercosporella herpotrichoides. In: Vereinigung österreichischer Pflanzenzüchter. Bericht über die Arbeitstagung 1987 der Arbeitsgemeinschaft der Saatzuchtleiter. Bundesanstalt für alpenländische Landwirtschaft Gumpenstein Österreich, 295-305

Maraite $H$, Delforge $C$, Meunier S, Tetelain A (1985) Distribution of MBC-resistance in Pseudocercosporella herpotrichoides on winter wheat in Belgium. Meded Fac Landbouwwet Rijksuniv Gent 50, 1173-1180

Mauler A, Fehrmann H (1987a) Erfassung der Anfälligkeit von Weizen gegnuber Pseudocercosporella herpotrichoides. I. Untersuchungen zur Pathogenität veschiedener Formen des Erregers. Z Pflanzenkr Pflanzenschutz 94, 630-636

Mauler A, Fehrmann H (1987b) Erfassung der Anfälligkeit von Weizen gegenuber Pseudocercosporella herpotrichoides. II. Zur Entwicklung eines Resistenztests an Jungpflanzen. Z Pflanzenkr Pflanzenschutz 94, 637-648

Moreau JM, Van Schingen JC, Maraite H (1989) Detection of Tapesia yallundae, the teleomorph of Pseudocercosporella herpotrichoides on wheat stubble in Belgium. Meded Fac Landbouwwet Rijksuniv Gent 54 (2b), 555-559

Moreau JM, Van Schingen JC, Maraite H (1990) Épidémiologie de Pseudocercosporella herpotrichoides var acuformis et var herpotrichoides sur froment d'hiver, démonstration d'un cycle secondaire. Meded Fac Landbouwwet Rijksuniv Gent 55 (3a), 889-898

Nirenberg $\mathrm{HI}$ (1981) Differenzierung der Erreger der Halmbruchkrankheit I. Morphologie. Z Pflanzenkr Pflanzenschutz 88, 241-248

Nirenberg $\mathrm{HI}$ (1984) Differenzierung der Erreger der Halmbruchkrankheit II. Physiologische Reaktionen in Kultur. $Z$ Pflanzenkr Pflanzenschutz 91, 225-235

Ponchet J (1959) La maladie du piétin-verse des céréales, Cercosporella herpotrichoides: importance agronomique, biologie, épiphytologie. Ann Epiphyt 10, 46-89

Poupard P, Cavelier N (1990) Variabilité intraspécifique de Pseudocercosporella herpotrichoides : caractérisation biochimique des différents types de souches par électrophorèse et modifi- cations des profils estérasiques en relation avec la sensibilité du champignon à certains fongicides inhibiteurs de la biosynthèse de l'ergostérol. $2^{\mathrm{e}}$ Cong Soc Fr Phytopathol Montpellier

Rapilly F, Laborie Y, Eschenbrenner P, Choisnel E, Lacroze $F$ (1979) La prévision du piétin-verse sur blé d'hiver Perspect Agric 23, 30-40

Sanders PL, De Waard MA, Loerakker WM (1986) Resistance to carbendazim in Pseudocercosporella herpotrichoides from Dutch wheat fields. Neth J Plant Pathol 92, 15-20

Sanderson FR, King AC (1988) Field occurrence of Tapesia yallundae, the teleomorph of Pseudocercosporella herpotrichoides Aust Plant Pathol 17, 20-21

Schreiber MT, Prillwitz HG (1985) Vorkommen von Pseudocercosporella-taxa an Wintergetreide in Rheinland-Pfalz. Nachrichtenbl Dtsch Pflanzenschutzdienst 37 (10), 145-150

Schreiber MT, Prillwitz HG (1986) Untersuchungen zur Pathogenität, Virulenz und Wirtspezifität von Pseudocercosporella-taxa an Wintergetreide. Nachrichtenbl Dtsch Pflanzenschutzdienst 38 (5), 65-71

Scott PR, Hollins TW (1980) Pathogenic variation in Pseudocercosporella herpotrichoides. Ann Appl Biol 94, 297-300

Scott PR, Hollins TW, Muir P (1975) Pathogenicity of Cercosporella herpotrichoides to wheat, barley, oats and rye. Trans Br Mycol Soc 65, 529538

Smith CM, Saunders DW, Allison DA, Johnson LEB, Labit B, Kendall SJ, Hollomon DW (1990) Immunodiagnostic assay for cereal eyespot : novel technology for disease detection. In: Pests and Diseases. Brighton Crop Protec Conf 7C17, 763-770

Unger JG (1989) Entwicklung und Erprobung eines ELISA (Enzyme-linked immunosorbent assay) zum Nachweis von Fusarium culmorum (WGSM) Sacc und Pseudocercosporella herpotrichoides (FRON) Deigh in Weizen. Doktorarbeit, Georg-August-Universität Göttingen, $134 p$

Van Schingen JC, Moreau JM, Maraite $H$, Meeus $P$ (1989) Maladies du pied sur froment d'hiver. Parasitica 45 (2-3), 49-55

Wallwork H (1987) A Tapesia-like teleomorph for the eyespot fungus Pseudocercosporella herpotrichoides. Aust Plant Pathol 16, 92-93

Wallwork H, Spooner B (1988) Tapesia yallundae - the teleomorph of Pseudocercosporella herpotrichoides. Trans Br Mycol Soc 91 (4), 703705

Weege D, Poupard P, Frei U, Lind V (1991) Electrophoretic identification of pathogen-specific proteins of foot rot-inducing fungi of wheat. Z Pflanzenkr Pflanzenschutz 98, 1-12 